\title{
Complex Activity Recognition using Context Driven Activity Theory in Home Environments
}

\author{
Saguna $^{1,2}$, Arkady Zaslavsky ${ }^{1,2}$, Dipanjan Chakraborty ${ }^{3}$ \\ ${ }^{1}$ Monash University, Melbourne, Victoria, Australia, \\ ${ }^{2}$ Lulea University of Technology, SE-971 87, Lulea, Sweden, \\ ${ }^{3}$ IBM Research, India Research Lab, New Delhi, India. \\ saguna.saguna@monash.edu, arkady.zaslavsky@ltu.se, cdipanjan@in.ibm.com
}

\begin{abstract}
This paper proposes a context driven activity theory (CDAT) and reasoning approach for recognition of concurrent and interleaved complex activities of daily living (ADL) which involves no training and minimal annotation during the setup phase. We develop and validate our CDAT using the novel complex activity recognition algorithm on two users for three weeks. The algorithm accuracy reaches $88.5 \%$ for concurrent and interleaved activities. The inferencing of complex activities is performed online and mapped onto situations in near real-time mode. The developed systems performance is analyzed and its behavior evaluated.
\end{abstract}

Keywords: Activity recognition, context-awareness, situations.

\section{Introduction}

Activity recognition in smart home environments focuses on the activities of daily living (ADL) such as cooking, housework, eating, office related work, grooming, etc. ADLs are the focus of several research areas such as health care, aged care, emergency, security and comfort [1-3]. The activities performed by human users are highly complex and multi-tasking comes naturally. Each ADL has more than one sub activity. For example, eating breakfast may involve sitting on chair, picking a knife, picking a fork, etc. We call these sub activities as atomic activities and define them as those unit level activities which cannot be broken down further given application semantics. Also, these atomic activities are performed as operations which are routinized [4]. Figure 1 shows typical ADLs performed by human users. These ADLs may interleave or occur concurrently when human users multi-task. Atomic activities may not always follow the same sequence within an ADL.

All human activity recognition problems somewhat follow a common approach. This includes the use of sensors, multi-sensor fusion, the segmentation of data with relation to time and space, feature extraction and selection, classification of activities, and the use of activity models to infer activities [5]. These models can be based on a number of techniques [5] such as machine learning and string-matching algorithms, human activity language and use of semantics. Activity models built for recognition of sequential activities do not perform well when activities are interleaved, occur concurrently or when sequence of atomic activities changes [3, 6, 7]. Also, the prima- 


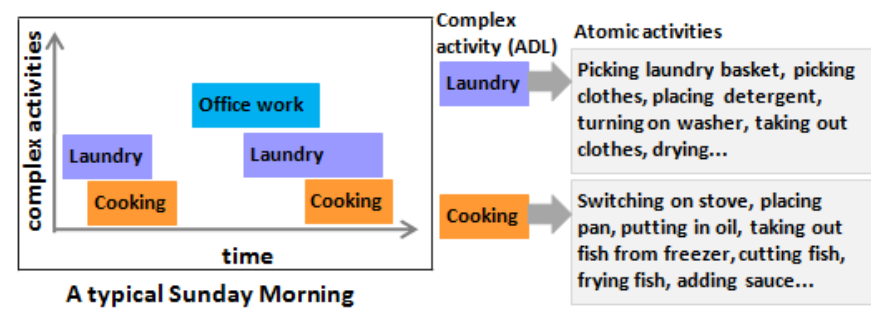

Fig. 1. ADLs performed by human users on a typical Sunday. Below we show atomic activities for two complex activities, Laundry and Cooking.

-ry focus is on atomic activities. State of the art $[3,8]$ uses probabilistic models or data mining techniques to infer sequential ADLs. The inference of sequential and varied order activities which are concurrent and interleaved is also recently been addressed in $[8,9]$. To summarize, these existing techniques suffer from several drawbacks. (1) Large amounts of data collection and data annotations are required for training of models, (2) Changes in sequence of ADLs affect activity recognition accuracy of trained models adversely, (3) Concurrent and interleaved ADLs are not accurately recognized as it is time consuming to train activity models for the different possible ways in which complex activities are interleaved or performed concurrently. In this paper, we focus on accurate and dependable recognition of complex ADLs ${ }^{1}$ by addressing these issues and using context information. The use of context information has been emphasized in [2] for activity recognition. Our contributions are three-fold. Firstly, we propose and develop a context-driven activity theory (CDAT) which is used in defining atomic and complex activities. Secondly, we develop a novel context-driven algorithm to infer complex concurrent and interleaved activities without the need for training data and minimal annotation only during setup. Finally, we develop a novel situation- and context-aware activity recognition system (SACAAR) which can be used by ubiquitous applications. We validate our system using extensive experimentation.

This paper is organized as follows: Firstly, we present the SACAAR approach for activity recognition which gives the theoretical design of our system. This is followed by the SACAAR system architecture and its prototype implementation. In the next section, the experimental results are presented which validate our proposed approach. We, then compare our SACAAR system with related work on complex activity recognition. Lastly, we give the conclusion and future work.

\section{SACAAR Approach to Activity Recognition}

This section presents our proposed approach to infer complex ADLs. In [10], activity information is considered to be a context. But in activity analysis domain, our aim is to facilitate the inference of activities using sensors and other context. In figure 2, we show how activities and situations are related in the activity analysis domain. Firstly,

\footnotetext{
${ }^{1}$ In the rest of the paper, ADLs are referred to as complex activities.
} 
context and sensors directly related to inferencing atomic activities are used, for example, one or more accelerometers are used for atomic activities like sitting and walking. Also, certain context information such as 'speed of user' may further validate whether user is walking or not. Further, a number of atomic activities together form a complex activity.

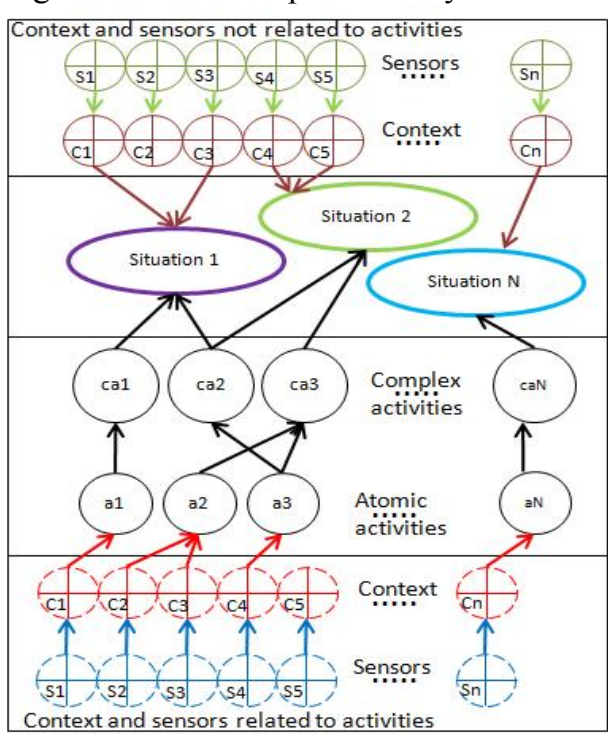

Fig. 2. Sensors(s), context(c), atomic activities(a), complex activities(ca), situations and their relationships. location sensor. This can help in activity inference since the user will perform only those activities which are linked to office room and not the kitchen.

We use the context spaces theory [11] to infer situations and then use this knowledge to further infer activities. A complex activity can be linked to more than one situation, for example, "eating lunch" can be performed in the "office room" or on a day off from work in the "dining room at home". Also, a complex activity can occur in time such that it traverses through more than one situation during its completion, for example, "getting ready and going to work" can belong to situations "leaving home", "out on the road" and "near office". The situations can be inferred from spatio-temporal information as well as other context information.

\subsection{Theoretical Design of SACAAR system}

In this section, we propose and investigate a context-driven activity theory (CDAT) where activity could be complex or atomic. We further develop a context and atomic activity reasoning approach to infer and reason about complex activities.

\footnotetext{
${ }^{2} \mathrm{http}: / /$ www.wordreference.com/definition/situation
}

Also, of interest here is the concept of role in activity inference Situations are defined as " $a$ set of circumstances in Human users in a day are part of a umber of situations. All activities that situations. We are referring to situations which are inferred by context related sensor data but other context for example, location, time, temperature, weather conditions, light on/off. By number of activities to be recognized. In a situation hierarchy, situations and subsituations exist and it is possible to dentify situations with a limited set of sensor and context information. For

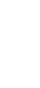

(1)

.

(1)

.

.


DEFINITION1: Atomic activity, $A$ is defined as activity which can be observed from a set of sensors, $\Sigma_{\mathrm{S}}=\left\{\mathrm{S}_{1}, \ldots, \mathrm{S}_{\mathrm{n}}\right\}$, where $\mathrm{n} \geq 1$, where the level of granularity can vary based on sensor deployment scenarios. For example,

Case 1: $A_{1}=$ "user is walking" inferred by sensor $s_{1}$ which is an accelerometer placed on the user's waist. Case 2: $A_{1}=$ "user is walking" inferred by sensors $s_{1}, s_{2}, s_{3}$ and $s_{4}$, where $s_{1}, s_{2}, s_{3}$ are three accelerometers placed on different parts of the body and $s_{4}$ is a GPS sensor placed on the user which provides individual body part movements and velocity information.

DEFINITION 2: Complex activity, $C A$ is a tuple $C A=\left(\gamma A, \rho C, A_{S} \vee^{3} C_{S}, A_{E} \vee C_{E}, T_{S}\right.$, $\mathrm{T}_{\mathrm{E}}, \mathrm{T}_{\mathrm{L}}$ ) where $\gamma \mathrm{A}$ is the sub-set of atomic activities from the complete set of atomic activities, $\Sigma_{\mathrm{A}}=\left\{\mathrm{A}_{1}, \ldots, \mathrm{A}_{\mathrm{n}}\right\}, \mathrm{n} \geq 1$ and $\rho \mathrm{C}$ is the sub-set of context information from the complete set of context information, $\Sigma_{C}=\left\{C_{1}, \ldots, C_{n}\right\}, n \geq 1$ which must occur for a complex activity $C A$ to occur (where the order within $\gamma \mathrm{A}$ is not important), $\left(\mathrm{A}_{\mathrm{S}}, \mathrm{A}_{\mathrm{E}}\right) \epsilon$ $\Sigma_{\mathrm{A}}$ and are the start and end atomic activities respectively, $\left(\mathrm{C}_{\mathrm{S}}, \mathrm{C}_{\mathrm{E}}\right) \in \Sigma_{\mathrm{C}}$ and are the start and end context information respectively. CA can have multiple start and end atomic activities as well as context information, $\mathrm{T}_{\mathrm{S}}$ and $\mathrm{T}_{\mathrm{E}}$ denote the start time and end time of a complex activity. $T_{L}=\left|T_{E}-T_{S}\right|$ is the complex activity lifespan and $\mathrm{T}_{\mathrm{Lmin}}<\mathrm{T}_{\mathrm{L}}<\mathrm{T}_{\mathrm{Lmax}}$ where $\left(\mathrm{T}_{\mathrm{Lmin}}, \mathrm{T}_{\mathrm{Lmax}}\right)$ gives the time range for a complex activity, for example,

Case 1: $\mathrm{CA}_{1}=$ "user is walking to bus stop" is inferred by atomic activity, $\mathrm{A}_{1}=$ user is walking and context information, $\mathrm{C}_{2}=$ user direction towards bus stop from home. Case 2: $\mathrm{CA}_{2}=$ "user is working on presentation at home" is inferred by atomic activities, $A_{2}=$ user is sitting, $A_{3}=$ user is using study desk, $A_{4}=$ user is detected near laptop, $\mathrm{A}_{5}=$ user is typing in Microsoft Powerpoint application file and context information, $\mathrm{C}_{3}=$ user location is home, $\mathrm{C}_{4}=$ study desk light is on. We further look at an example of concurrent and interleaved activities. Case 3: A user performs the following activities concurrently and interleaved in time. $\mathrm{CA}_{3}=$ "user is reading/writing a document on his/her laptop in room at office", $\mathrm{CA}_{4}=$ "user is browsing the Internet for research articles", $\mathrm{CA}_{5}=$ "user is drinking coffee", $\mathrm{CA}_{6}=$ "user is chatting on IM with friend". We can further perform operations on complex activities such as unions to create complex activities such as $\left(\mathrm{CA}_{3} \cup \mathrm{CA}_{4} \cup \mathrm{CA}_{5}\right)$.

We do not consider order of atomic activities while performing complex activity inference though if required, it can be accommodated in Definition 2 by changing $\gamma \mathrm{A}$ to a fixed-ordered list. Fixed order of atomic activities is important in industry environments or workshop settings, in real life it varies considerably.

Context and Atomic Activity Reasoning to Infer Complex Activities: To infer complex activities we propose reasoning of context and atomic activity information. Each complex activity has a list of atomic activities, $\gamma \mathrm{A}$ and a list of context information, $\rho \mathrm{C}$ as mentioned in Definition 2. Table 1 gives some examples to explain our reasoning approach. Each atomic activity, $A_{i}$ and context information, $C_{i}$ is assigned a particular weight, $w_{C A_{k}}^{A_{i}}$ and $w_{C A_{k}}^{C_{i}}$ respectively, corresponding to its importance in relation to the occurrence of a complex activity $\mathrm{CA}_{\mathrm{k}}$. The sum of all the weights, $\omega_{C A_{k}}$ for each $\mathrm{CA}_{k}$ is 1 . If $\mathrm{A}_{\mathrm{i}}$ or $\mathrm{C}_{\mathrm{i}}$ do not occur for a particular $\mathrm{CA}_{k}$ then $w_{C A_{k}}^{A_{i}}=0$ and $w_{C A_{k}}^{C_{i}}=0$. The sum of all the weights, $\omega_{C A_{k}}$ for all occurring atomic

\footnotetext{
${ }^{3} \vee$ implies the OR operation.
} 
activities and context information needs to be above a threshold, $\omega_{C A_{k}}^{T}$ in order for $\mathrm{CA}_{k}$ to occur successfully. If the sum of weights, $\omega_{C A_{k}}$ is less than the threshold, $\omega_{C A_{k}}^{T}$ then 1) it implies that the activity was started but abandoned in between, 2) it implies that the core set of atomic activities and context information for that particular $\mathrm{CA}_{k}$ did not occur. Thus,

where, $0 \leq \omega \leq 1$ and

$$
\omega_{C A_{k}}=\sum_{i=1}^{N} w_{C A_{k}}^{A_{i}}+\sum_{i=1}^{n} w_{C A_{k}}^{C_{i}}
$$

and

$$
\sum_{i=1}^{N} w_{C A_{k}}^{A_{i}}=\left(w_{C A_{k}}^{A_{1}}+\cdots+w_{C A_{k}}^{A_{N}}\right)
$$

$$
\sum_{i=1}^{N} w_{C A_{k}}^{C_{i}}=\left(w_{C A_{k}}^{C_{1}}+\cdots+w_{C A_{k}}^{C_{N}}\right)
$$

and $w_{C A_{k}}^{A_{i}}=0$ and $w_{C A_{k}}^{C_{i}}=0$, if $\mathrm{A}_{\mathrm{i}}$ and $\mathrm{C}_{\mathrm{i}}$ do not occur for $\mathrm{CA}_{k}$ and

$$
\omega_{C A_{k}} \geq \omega_{C A_{k}}^{T}
$$

for any $\mathrm{CA}_{k}$ to have occurred successfully. We further demonstrate the use of weights which helps in complex activity recognition by checking the occurrence of key atomic activities using the following examples from table 1 .

Example 1: $\mathrm{CA}_{l}=$ Cooking omelette for breakfast in kitchen is a complex activity as shown in table I. We use our CDAT along with our reasoning approach to define and infer $\mathrm{CA}_{l}$. We define the $\mathrm{CA}_{l}=\left(\gamma \mathrm{A}, \rho \mathrm{C}, \mathrm{A}_{\mathrm{S}} \vee \mathrm{C}_{\mathrm{S}}, \mathrm{A}_{\mathrm{E}} \vee \mathrm{C}_{\mathrm{E}}, \mathrm{T}_{\mathrm{S}}, \mathrm{T}_{\mathrm{E}}, \mathrm{T}_{\mathrm{L}}\right)$ tuple based

\begin{tabular}{|c|c|c|c|c|c|c|c|c|c|}
\hline $\begin{array}{c}C A_{k} \\
\left(\omega_{C A_{k}}^{T}\right)\end{array}$ & $\gamma \mathbf{A}\left(w_{C A_{k}}^{A_{i}}\right)$ & $\rho \mathrm{C}\left(w_{C A_{k}}^{C_{i}}\right)$ & $\begin{array}{c}\text { Core } \gamma A \\
\text { and } \rho C\end{array}$ & $\begin{array}{l}\mathbf{A}_{\mathbf{S}} \\
, \mathbf{C}_{\mathrm{S}}\end{array}$ & $\begin{array}{l}\mathbf{A}_{\mathbf{E}} \\
\mathbf{C}_{\mathbf{E}}\end{array}$ & $\mathbf{T}_{\mathrm{S}}$ & $\mathbf{T}_{\mathbf{E}}$ & $\begin{array}{r}\text { Time } \\
\text { taken } \\
\text { (mins) }\end{array}$ & $\begin{array}{c}\mathrm{T}_{\mathrm{L}} \\
\text { range } \\
\text { (mins) }\end{array}$ \\
\hline $\begin{array}{l}\text { Cooking } \\
\text { omelette } \\
\text { for } \\
\text { breakfast } \\
\text { in kitchen } \\
(0.89)\end{array}$ & $\begin{array}{l}\mathrm{A}_{1}: \operatorname{standing}(0.01), \\
\mathrm{A}_{2}: \text { walking }(0.01), \\
\mathrm{A}_{3}: \text { fridge }(0.02), \\
\mathrm{A}_{4}: \operatorname{plate}(0.02), \\
\mathrm{A}_{5}: \operatorname{eggs}(0.16), \\
\mathrm{A}_{6}: \text { frypan }(0.14), \\
\mathrm{A}_{7}: \operatorname{vegetable} \\
\text { drawer(0.02), } \\
\mathrm{A}_{8}: \operatorname{bowl}(0.12), \\
\mathrm{A}_{9}: \operatorname{whisker}(0.12)\end{array}$ & $\begin{array}{l}\mathrm{C}_{1}: \text { in home } \\
\text { kitchen } \\
(0.12) \text {, } \\
\mathrm{C}_{2}: \text { morning } \\
(0.12), \\
\mathrm{C}_{3}: \text { kitchen } \\
\text { light } \\
\text { on }(0.03) \text {, } \\
\mathrm{C}_{4}: \text { stove } \\
\text { on }(0.12)\end{array}$ & $\begin{array}{l}\text { Core } \gamma \mathrm{A}= \\
\left(\mathrm{A}_{5}, \mathrm{~A}_{6},\right. \\
\left.\mathrm{A}_{8}, \mathrm{~A}_{9}\right) \\
\text { and } \\
\text { Core } \rho \mathrm{C}= \\
\left(\mathrm{C}_{1}, \mathrm{C}_{2}, \mathrm{C}_{4}\right)\end{array}$ & $\begin{array}{l}\mathrm{C}_{1}, \\
\mathrm{~A}_{5}, \\
\mathrm{~A}_{3}, \\
\mathrm{~A}_{6}, \\
\mathrm{~A}_{10}, \\
\mathrm{~A}_{9}\end{array}$ & $\begin{array}{l}\neg \mathrm{C}_{3}, \\
\mathrm{~A}_{4}, \\
\neg \mathrm{C}_{4}\end{array}$ & 07:06 & $07: 22$ & 16 & $10-20$ \\
\hline $\begin{array}{l}\text { Drinking } \\
\text { coffee in } \\
\text { office } \\
\text { room } \\
(0.68)\end{array}$ & $\begin{array}{l}\mathrm{A}_{1}: \text { coffeemug } \\
(0.34), \\
\mathrm{A}_{2}: \operatorname{sitting}(0.16) \\
\mathrm{A}_{2}: \text { walking }(0.16)\end{array}$ & $\begin{array}{l}\mathrm{C}_{1} \text { :in office } \\
\operatorname{room}(0.34)\end{array}$ & $\begin{array}{l}\text { Core } \gamma \mathrm{A}= \\
\left(\mathrm{A}_{1}\right) \text { and } \\
\text { Core } \rho \mathrm{C}= \\
\left(\mathrm{C}_{1}\right)\end{array}$ & $\begin{array}{l}\text { coffee } \\
\text { mug }\end{array}$ & $\begin{array}{l}\neg \text { coff } \\
\text { ee } \\
\text { mug }\end{array}$ & $18: 15$ & $18: 45$ & 30 & $20-45$ \\
\hline $\begin{array}{l}\text { Working } \\
\text { on a doc } \\
\text { in office } \\
\text { room } \\
(0.68)\end{array}$ & $\begin{array}{l}\mathrm{A}_{1}: \operatorname{sitting}(0.16) \\
\mathrm{A}_{2}: \text { typing word } \\
\operatorname{doc}(0.34)\end{array}$ & $\begin{array}{l}\mathrm{C}_{1} \text { : in office } \\
\text { room }(0.16), \\
\mathrm{C}_{2}: \text { usingdesk } \\
(0.34)\end{array}$ & $\begin{array}{l}\text { Core } \gamma \mathrm{A}= \\
\left(\mathrm{A}_{2}\right) \text { and } \\
\text { Core } \rho \mathrm{C}= \\
\left(\mathrm{C}_{2}\right)\end{array}$ & $\begin{array}{l}\text { word } \\
\text { doc }\end{array}$ & $\begin{array}{l}\neg \text { wor } \\
\text { d doc }\end{array}$ & $10: 10$ & $11: 30$ & 80 & $10-60$ \\
\hline
\end{tabular}
on domain knowledge in a way that it is straightforward for users to define the tuple themselves by using the type of atomic activities and context information available.

Table 1. Complex Activity Examples. 
The assignment of weights is based on the importance of each $A_{i}$ and $C_{i}$ for a corresponding $\mathrm{CA}_{1}$, for example, here the highest weight is assigned to $\mathrm{A}_{5}=0.16$ followed by $\mathrm{A}_{6}=0.14$ which are very important atomic activities for cooking an omelette. Also, $\mathrm{C}_{1}, \mathrm{C}_{2}$ and $\mathrm{C}_{4}$ are assigned weights of 0.12 as they are all of equal importance in the process of performing $\mathrm{CA}_{l}$ while $\mathrm{C}_{3}$ may or may not be switched on depending on time of day. In this case, $\omega_{C A_{k}}$ should be greater than the threshold $\omega_{C A_{k}}^{T}=0.89$ for $\mathrm{CA}_{1}$ to have successfully occurred. Also, temporal context is part of the complete set of context information, $\Sigma_{\mathrm{C}}$. $\neg \mathrm{A}$ and $\neg \mathrm{C}$ represents non occurrence of atomic activity and context information.

Similarly, for complex activities $\mathrm{CA}_{2}=$ Drinking coffee in lounge and $\mathrm{CA}_{3}=$ Working on a document in office can be reasoned and inferred as shown in table 1 . We highlight that uncertain context or sensor information can also be dealt with by taking the probabilities of occurrence of atomic activities and multiplying with their assigned weights in Equation 1.

\section{SACAAR System Architecture}

The SACAAR system architecture consists of two layers as shown in figure 3. It can be deployed in different application domains which require complex activity recognition. The sensory layer of the system can handle different types of sensor information which is required to infer atomic activities. For example, accelerometers to infer body motion such as sitting, walking, standing, jogging, RFID reader and tags for object interaction such as picking a cup, picking a pan, touch sensors for opening a cupboard, opening a door, physiological sensors for mood and stress levels. Virtual sensors can include modules of code which gather device activity, browser activity and music player activity from a user's devices such as computers, laptops and smart phones. We infer three types of context information. Firstly context information that helps in improving activity inference for example, if sensor 1(accelerometer) returns atomic activity walking, the confidence in this atomic activity can be increased by context information from sensor 2(GPS) which provides the speed of the user. Secondly, context information which helps in inferring complex activities for example, in the above case sensor 2(GPS) can also give direction of the user between 2 points $\mathrm{A}$ and $\mathrm{B}$. This helps in inferring complex activity such as user walking towards point B. Lastly, context information can be used to infer situations. The activity inference layer is used to infer activities and it consists of the following components:

Atomic Activity Inference Module:

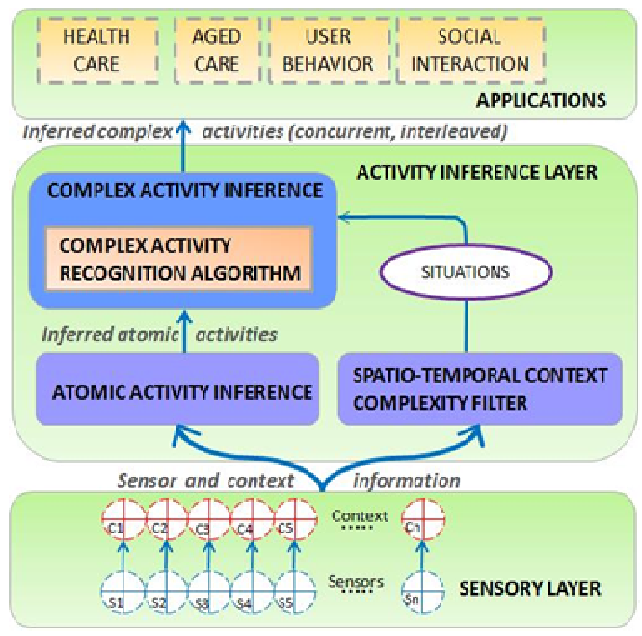

Fig. 3. SACAAR architecture. 
In AMOD, atomic activities are inferred. Body motion related atomic activities such as sitting, standing, walking, etc are inferred using decision trees [12]. A weighted voting sliding window mechanism is used to infer RFID object usage. A similar sliding window weighted voting mechanism is also applied to device and browser activity. Other context information such as speed and direction are used to ascertain activities as well.

Spatio-temporal Context Complexity Filter: Context information from the sensory

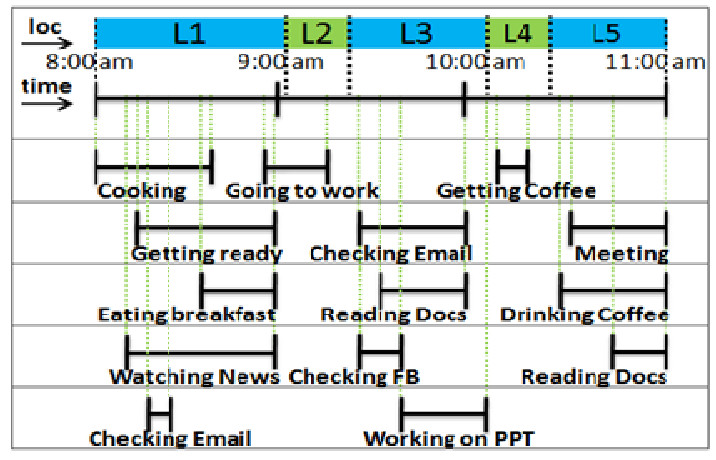

Fig. 4. Multiple complex activities inferred from 8:00 am to 11:00 am on a weekday (Day 12 for Subject 1 from our experiments) with locations $\mathrm{L} 1=$ Home, L2 $=$ Outdoor/on the road, L3=Office room, L4=Office kitchen and L5=Meeting room. The labels for activities are generalized in some cases for brevity, for example cooking is a label for "cooking omelette for breakfast" and checking FB is social networking layer is used by $\mathrm{CCF}$ for inferencing situations. This information is used in two ways by our SACAAR system architecture. Firstly, context information is used to infer situations. Activities always belong to some situation and linking them can help in inference of activities as well as enhance the richness of the inferred complex activity. These linkages can be created at run time and new activities can be added to situations in SACAAR system. We initially created situations and link our pre-defined complex activities to these situations during the setup phase. We then provided a mechanism to add activities to new situations at run time, if required. Secondly, when the links between activities and situations are known to be fixed, it can help to reduce the number of complex activities to look for during the inference process by inferring the situations first. Such situation inference can help in activity recognition by reducing battery consumption of certain sensors which are being polled to infer atomic activities which do not belong to the current situation.

Complex Activity Inference Module: The CAIM is used to fuse together different sources of atomic activities and combine them to infer complex activities with the help of the situation and activity linkages provided by the CCF. The complex activity recognition algorithm (CARALGO) is then used for inference of complex activities which can be concurrent and interleaved and is given in table 2. Figure 4 shows a snapshot of the inferred concurrent and interleaved complex activities from day 12 of our experiment 2 using subject 1 (see section 5). Some labels are generalized for brevity. It works by taking atomic activities, context information and situations as input and outputs the complex activities. The algorithm consists of finding the start atomic activity and then setting a time window of the size of the lifespan $\mathrm{T}_{\mathrm{L}}$ for each matched $A_{S} \vee C_{S}$ belonging to a $C A_{k}$. We then look for matching $\gamma \mathrm{A}, \rho C$ and $A_{E} \vee C_{E}$ within the time window for each $\mathrm{CA}_{\mathrm{k}}$. We compute the $\omega_{C A_{k}}$ using equation (1) and then check against $\omega_{C A_{k}}^{T}$ as shown in equation (4). If the condition is matched $\mathrm{CA}_{k}$ is inferred successfully. 
Table 2. Complex Activity Recognition Algorithm (CARALGO).

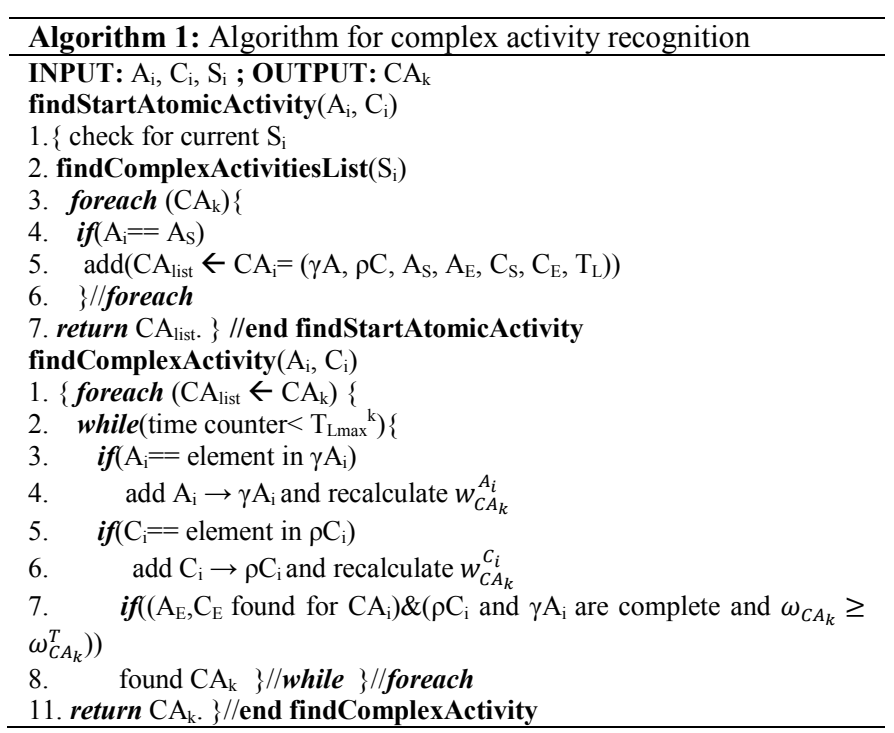

All time windows run in parallel and all incoming $\mathrm{A}_{i}$ and $\mathrm{C}_{i}$ for each $\mathrm{CA}_{k}$ are added to them till a successful match is found. The weights are added at runtime after each addition. We assign weights currently using domain knowledge but in the future we are looking at techniques to assign weights automatically.

\section{SACAAR System Test-bed and Prototype Implementation}

We have built a test-bed comprising of several sensors. We place either Mulle v3 sensor $^{4}$ (accelerometer) or the Android Phone with its inbuilt accelerometer on the user's waist to detect body motion using decision trees. We chose the waist of the user as the most appropriate position [2]. A Bluetooth RFID reader was used. It was placed on the user's wrist to detect LF passive RFID tagged objects within a distance of $5-10 \mathrm{~cm}$. Each tag is labeled in terms of an activity, and the RFID readings are inferred as atomic activities. The device and browser activity are inferred using a $\mathrm{C}++$ TimeTracker $^{5}$ tool available openly on sourceforge. We extended this tool to incorporate it in our test-bed. The user's activity on the mobile phone is inferred using a Java based code built by us specific to the Android platform. Location information is collected using GPS, Wi-fi positioning and RFID tags. We use the inbuilt GPS on an Android phone to gather the location, speed and weather information. We will provide the data and prototype online to share with those interested. Further details on test-bed can also be found in [13].

\footnotetext{
${ }^{4} \mathrm{http}: / /$ www.eistec.se/

${ }^{5} \mathrm{http}: / /$ tracker.sourceforge.net/
} 


\section{Experimental Results}

We use our test-bed for testing and validating our proposed approach to recognize complex activities which are concurrent and interleaved. We conducted extensive experimentation and we present the results in this section. We perform experiments on two subjects for the duration of 21 days with an average of seven hours daily. The experiments were performed usually in the time range of 8:00 am to 12:00 pm and from 1:30 pm to 5:30 pm. We identified 16 complex activities (listed in table 3 ) and used our CDAT to define them. These definitions were stored in the SACAAR system. We gave our subjects an Android phone to record the activities manually which involved just adding a count for each occurrence of a complex activity in the corresponding hour. The user was asked to keep this record simply for measuring the accuracy of our algorithm. This information was not used for any annotation or training purpose. We show the accuracy of our algorithm for both subjects in table 3 . Subject one performed all activities while subject two performed only 13 activities out of the 16 . Our algorithm performed with an overall accuracy of $88.5 \%$.

We performed online inferencing and users were given complete freedom to perform the previously defined complex activities in any interleaved and concurrent manner. We allowed users to change sequence of atomic activities as well. It is important to note that our approach does not require training data to create activity models. It only uses domain knowledge to define complex activities and create links to atomic activities. The activities in table 3 with format <activity@loc> always occurred at the same location while activities with format $<$ activity $>$ occurred at both home and office. Figure 5(a) shows SACAAR running on Android Phone with online inferencing. The snapshot shows atomic activities and context information with their corresponding weights which is followed by the inferred complex activity. To the best of our knowledge this is the first such work to infer complex activities using CDAT.

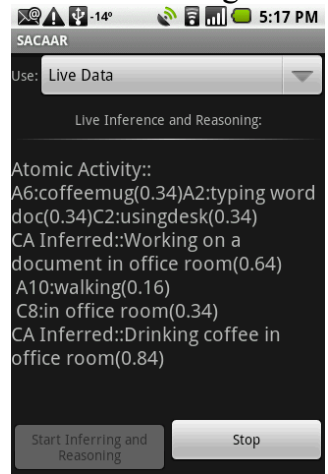

$5(\mathrm{a})$

\begin{tabular}{c|ccccccccccc}
\hline CA(k) & 2 & 3 & 4 & 5 & 6 & 9 & 10 & 11 & 12 & 13 & 14 \\
\hline 2 & 0 & 6 & 12 & 15 & 5 & 0 & 2 & 0 & 5 & 3 & 0 \\
3 & 6 & 0 & 15 & 14 & 5 & 0 & 1 & 0 & 2 & 4 & 0 \\
4 & 12 & 15 & 0 & 28 & 30 & 5 & 1 & 0 & 11 & 0 & 2 \\
5 & 15 & 14 & 28 & 0 & 45 & 17 & 23 & 25 & 74 & 90 & 110 \\
6 & 5 & 5 & 30 & 45 & 0 & 5 & 7 & 3 & 29 & 100 & 43 \\
9 & 0 & 0 & 5 & 17 & 5 & 0 & 24 & 11 & 45 & 10 & 76 \\
10 & 2 & 1 & 1 & 23 & 7 & 24 & 0 & 27 & 19 & 5 & 63 \\
11 & 0 & 0 & 0 & 25 & 3 & 11 & 27 & 0 & 14 & 0 & 0 \\
12 & 5 & 2 & 11 & 74 & 29 & 45 & 19 & 0 & 0 & 9 & 133 \\
13 & 3 & 4 & 0 & 90 & 100 & 10 & 5 & 14 & 9 & 0 & 5 \\
14 & 0 & 0 & 2 & 110 & 43 & 76 & 63 & 0 & 133 & 5 & 0 \\
\hline
\end{tabular}

$5(\mathrm{~b})$

Fig 5. (a) SACAAR system running on Android Phone showing live inferencing of atomic and complex activities. (b) Matrix showing number of instances of interleaved and concurrent complex activities for 11 activities from table 3 .

Figure 5(b) shows the activity pairs which had maximum concurrency and interleaving. We observe that $\left(\mathrm{CA}_{14}, \mathrm{CA}_{12}\right)$ and $\left(\mathrm{CA}_{14}, \mathrm{CA}_{5}\right)$ have the highest two values. $\mathrm{CA}_{16}$ and $\mathrm{CA}_{15}$ were the least interleaved activities with any other $\mathrm{CA}_{\mathrm{k}}$ (not 
Table 3. Output instances of complex activity from subject one (U1) and subject two (U2) with the individual accuracy values. The complex activities, CA(k) comprised of the following: $\mathrm{CA}(1)=$ "Getting ready@home”, $\mathrm{CA}(2)=$ "Cooking omelettefor breakfast@ @ome”, CA(3) = "Eating breakfast@ @ome", CA(4)="Watching news", CA(5)="Checking email"; CA(6) $=$ "Social networking", CA(7) = "Going to work@ outdoor", CA $(8)=$ "Getting coffee", $\mathrm{CA}(9)=$ "Drinking coffee", $\mathrm{CA}(10)=$ "Working on presentation"; $\mathrm{CA}(11)=$ "Meeting @ office", $\mathrm{CA}(12)=$ "Read/Write docs", $\mathrm{CA}(13)=$ "Watching videos online", $\mathrm{CA}(14)=$ "Searching research articles", CA(15) = "Jogging in the gym @ office”, CA(16)="Walking in city@mall”.

\begin{tabular}{|c|c|c|c|c|c|c|}
\hline \multirow{2}{*}{$\begin{array}{l}\text { Complex } \\
\text { Activity } \mathbf{C A}_{k}\end{array}$} & \multicolumn{2}{|c|}{$\begin{array}{l}\text { Correctly } \\
\text { Inferred }\end{array}$} & \multicolumn{2}{|c|}{$\begin{array}{l}\text { Incorrectly inferred } \\
\text { or abandoned }\end{array}$} & \multicolumn{2}{|c|}{ Accuracy (\%) } \\
\hline & U1 & U2 & U1 & U2 & U1 & U2 \\
\hline 1 & 16 & 12 & 3 & 2 & 84 & 86 \\
\hline 2 & 8 & 10 & 3 & 1 & 73 & 91 \\
\hline 3 & 12 & 12 & 2 & 2 & 86 & 86 \\
\hline 4 & 18 & 16 & 2 & 4 & 90 & 80 \\
\hline 5 & 100 & 74 & 29 & 3 & 78 & 96 \\
\hline 6 & 60 & 45 & 10 & 3 & 86 & 94 \\
\hline 7 & 12 & 14 & 6 & 1 & 67 & 93 \\
\hline 8 & 30 & - & 6 & - & 83 & - \\
\hline 9 & 25 & - & 8 & - & 76 & - \\
\hline 10 & 20 & 33 & 3 & 3 & 87 & 92 \\
\hline 11 & 16 & 22 & 2 & 9 & 89 & 71 \\
\hline 12 & 75 & 85 & 7 & 6 & 91 & 93 \\
\hline 13 & 70 & 60 & 6 & 4 & 92 & 94 \\
\hline 14 & 98 & 100 & 4 & 11 & 96 & 90 \\
\hline 15 & 8 & - & 0 & - & 100 & - \\
\hline 16 & 5 & 4 & 0 & 1 & 100 & 80 \\
\hline Total & 569 & 487 & 95 & 50 & 86 & 91 \\
\hline
\end{tabular}

Table 4. Complex activity linked to situations (' $X$ ' denotes occurrence and '-' denotes not occurring) ( $\mathrm{S} 1$ = Afternoon in office, $\mathrm{S} 2=$ Morning in city, S3 = Evening at home, S4 = Early morning at home).

\begin{tabular}{|l|c|c|c|c|}
\hline \multicolumn{1}{|c|}{$\begin{array}{c}\text { Situations(S) } \rightarrow \\
\text { Complex Activity } \downarrow\end{array}$} & S1 & S2 & S3 & S4 \\
\hline Searching research articles & - & - & - & X \\
\hline Watching online videos & X & - & - & - \\
\hline Cooking omelette & - & - & X & - \\
\hline Jogging in gym & X & - & - & - \\
\hline Walking in city & - & X & - & - \\
\hline
\end{tabular}


shown in figure 5b). It is observed that mostly activities in the morning and in the office are most interleaved and concurrent. We highlight that interleaving of complex activities is observed from the interleaving atomic activities belonging to different complex activities. Concurrency of complex activities is observed when complex activities occur within same time periods. Experimentation showed that the key to improving accuracy of inferring activities using CARALGO is the complex activity lifespan. We inferred situations linked to spatio-temporal context such as "at home in the morning", "outdoor early morning", "outdoor in the afternoon", "outdoor in the evening", "at office in the day", "in the kitchen in the morning", etc. Activities occurring in these situations were linked to them. This helped in the inference of activities as well as we ascertained which activities belonged to which situations. Any activity from our defined set when occurring in a situation not linked to it previously was recorded and the system was notified for later addition or deletion. We had 12 complex activities that were notified to be added to 5 situations which were not linked previously. We show in table 4,5 such activities linking to 4 situations. We do not show all situations that were defined at setup and their activity links here due to lack of space. We plan to share the data collected as well as the activity model used for inferencing in this experiment with research community.

\section{Related Work on Complex Activity Recognition}

In $[1,2]$, activity recognition involves collecting and labeling large datasets along with training models using the collected data. SACAAR does not require training data for complex activity inference and only uses minimal domain knowledge to define activities. By using CDAT atomic and complex activities can be defined easily. Also, in existing research, once the activity models are created any addition or change in the steps of activities will require collecting data and training the model again while SACAAR helps in eliminating this tedious procedure. Another advantage of SACAAR is that it is used to infer complex activities which are interleaved and concurrent. Data mining techniques applied to concurrent and interleaved activities in [8] are interesting as they help in searching emerging patterns using frequent itemset mining and find abnormally growing patterns but we differ from this work fundamentally as we use our CDAT and additional context information as well as a reasoning approach to infer activities.

In [9], activities are discovered using a mining method called discontinuous variedorder mining and then clustering is performed to identify activities. For the inference of activities voted hidden Markov model is used and the relative frequencies from sample data are used for training. This would still require observing individuals for weeks to create clusters of activities and then human input to identify the clusters. We highlight that CDAT is still required to define activities. We differ from [9] as our approach uses additional context information as well as a reasoning technique which improves accuracy and helps in identifying key atomic activities for complex activities. Our work also differs from [8,9]. We use wearable sensors which can facilitate the process of linking activities to users and in the future our SACAAR system can be used to recognize complex activities involving multiple users. 


\section{Conclusion and Future Work}

In this paper, we propose, develop and evaluate SACAAR system which infers atomic and complex activities by mapping them onto situations. Firstly, we propose and develop a novel system which uses multiple sensors and context information to infer atomic and complex activities. Secondly, we presented our context-driven activity theory (CDAT) and complex activity recognition algorithm (CARALGO) which infers complex activities that are interleaved and concurrent in nature. Thirdly, we perform activity and context reasoning to infer complex activities. A test-bed and prototype are built to validate SACAAR and CARALGO. The experimental results show that activity inference with considerably high accuracy was achieved with minimal domain knowledge and without any training at complex activity level. Future work will extend our context-driven CDAT, reasoning approach and the SACAAR system to infer complex activities involving multiple users performing complex activities in same situations.

\section{References}

[1] M. Philipose, K. P. Fishkin, M. Perkowitz, D. J. Patterson, D. Fox, H. Kautz, and D. Hahnel, "Inferring activities from interactions with objects," Pervasive Computing, IEEE, vol. 3, pp. 50-57, 2004.

[2] T. Choudhury, S. Consolvo, B. Harrison, J. Hightower, A. LaMarca, L. LeGrand, A. Rahimi, A. Rea, G. Bordello, B. Hemingway, P. Klasnja, K. Koscher, J. A. Landay, J. Lester, D. Wyatt, and D. Haehnel, "The Mobile Sensing Platform: An Embedded Activity Recognition System," Pervasive Computing, IEEE, vol. 7, pp. 32-41, 2008.

[3] A. Ferscha, F. Mattern, E. Tapia, S. Intille, and K. Larson, "Activity Recognition in the Home Using Simple and Ubiquitous Sensors," in Pervasive Computing. vol. 3001: Springer, 2004, pp. 158-175.

[4] V. Kaptelinin, B. Nardi, and C. Macaulay, "Methods \& tools: The activity checklist: a tool for representing the "space" of context," interactions, vol. 6, pp. 27-39, 1999.

[5] G.-Z. Yang, B. Lo, and S. Thiemjarus, Body Sensor Networks: Springer London, 2006.

[6] F. Albinali, N. Davies, and A. Friday, "Structural Learning of Activities from Sparse Datasets," in Pervasive Computing and Communications, Fifth Annual IEEE International Conference on, 2007, pp. 221-228.

[7] N. Davies, D. P. Siewiorek, and R. Sukthankar, "Activity-Based Computing," Pervasive Computing, IEEE, vol. 7, pp. 20-21, 2008

[8] G. Tao, W. Zhanqing, T. Xianping, P. Hung Keng, and L. Jian, "epSICAR: An Emerging Patterns based approach to sequential, interleaved and Concurrent Activity Recognition," in Pervasive Computing and Communications, IEEE International Conference on, 2009, pp. 1-9.

[9] P. Rashidi, D. Cook, L. Holder, and M. Schmitter-Edgecombe, "Discovering Activities to Recognize and Track in a Smart Environment," Knowledge and Data Engineering, IEEE Transactions on, vol. 23, no. 4, pp. 527-539, April 2011.

[10] A. K. Dey, "Providing architectural support for building context-aware applications," PhD Thesis, Georgia Institute of Technology, 2000, p. 240.

[11] A. Padovitz, S. W. Loke, and A. Zaslavsky, "Towards a Theory of Context Spaces," Proceedings of the Second IEEE Annual Conference on Pervasive Computing and Communications Workshops, 2004.

[12] L. Bao and S. Intille, "Activity Recognition from User-Annotated Acceleration Data," in Proc. Pervasive, Vienna, Austria, 2004, pp. 1-17.

[13] S. Saguna, A. Zaslavsky, and D. Chakraborty, "CrysP: Multi-Faceted Activity-Infused Presence in Emerging Social Networks," in ruSMART 2010. vol 6294. LNCS: Springer, 2010. 